\title{
SYNCHRONIZATION IN COUPLED NEURAL NETWORK WITH INHIBITORY COUPLING
}

\author{
Andrey Andreev \\ Neuroscience and Cognivite Technology Laboratory \\ Center for Technologies in Robotics and \\ Mechatronics Components \\ Innopolis University \\ Russia \\ a.andreev@innopolis.ru
}

\author{
Vladimir Maksimenko \\ Neuroscience and Cognivite Technology Laboratory \\ Center for Technologies in Robotics and \\ Mechatronics Components \\ Innopolis University \\ Russia \\ v.maximenko@innopolis.ru
}

Article history:

Received 15.11.2019, Accepted 26.12.2019

\begin{abstract}
A theoretical model of a network of neuron-like elements was constructed. The network included several subnetworks. The first subnetwork was used to translate a constant-amplitude signal into a spike sequence (conversion of amplitude to frequency). A similar process occurs in the brain when perceiving visual information. With an increase in the flow of information, the generation frequency of the neural ensemble participating in the processing increases. Further, the first subnetwork transmitted excitation to two large interconnected subnetworks. These subnetworks simulated the dynamics of the cortical neuronal populations. It was shown that in the presence of inhibitory coupling, the neuronal ensembles demonstrate antiphase dynamics. Various connectivity topologies and various types of neuron-like oscillators were investigated. We compare the results obtained in a discrete neuron model (Rulkov model) and a continuous-time model (Hodgkin-Huxley). It is shown that in the case of a discrete neuron model, the periodic dynamics is manifested in the alternate excitation of various neural ensembles. In the case of the continuous-time model, periodic modulation of the synchronization index of neural ensembles is observed.
\end{abstract}

\section{Key words}

Neural network, synchronization, Hodgkin-Huxley model, Rulkov map.

\section{Introduction}

Nowadays, an understanding of processes of interaction between different brain areas under cognition is an important task. Many scientists use methods of networks theory to analyse the processes [Palmeri and Gauthier, 2004; Haenschel et al., 2005; Cavanna and Monaco, 2009] based on both analysis of experimental data (electrical or magnetical multichannel recordings) [Maksimenko et al., 2018b; Campbell, 2009; Buzsáki, 2004; Chholak et al., 2019; Buzsáki and Draguhn, 2004; Maksimenko et al., 2018a] and numerical simulation of neurons interaction [Valencia et al., 2008; Andreev et al., 2017; Klinshov et al., 2015; Klinshov et al., 2014].

An idea of creating mathematical models describing some features of processes of interaction is actual [Yun et al., 2012]. Investigation of cognitive interaction is in line with the construction of models of nonlinear networks with complex topology [Boccaletti et al., 2006; Maslennikov and Nekorkin, 2017]. It was shown that topology is the main factor determining the emergence, stability, and propagation of synchronous states in complex networks [Moreno and Pacheco, 2004]. For example, in a number of works it was shown that the structure of freely scalable networks, which include, in particular, the neural network of the brain [Bullmore and Sporns, 2009] is more resistant to external influences and contributes to faster network synchronization [GómezGardenes et al., 2011; Gómez-Gardenes et al., 2007; Andreev et al., 2019].

Synchronization processes within complex networks have been closely studied for a long time, but processes of the interaction between networks of dynamic elements are still very poorly studied [Gao et al., 2014]. Nevertheless, interest in this problem has increased sharply in recent years, and the attention of researchers is shifting to the study of the processes of competition and synchronization between interacting networks, which is associated with the practical possibilities of describing 
many processes of real systems [Jiang and Perc, 2013; Klinshov et al., 2014; Bolotov et al., 2016].

In this paper, we numerically study a neural network of several subnetworks. The first subnetwork receives an external stimulus and transmites excitation to two large interconnected subnetworks. We show that in the presence of inhibitory coupling, the neuronal ensembles demonstrate antiphase dynamics. Various connectivity topologies ("all-to-all", "small-world" and completely random) and various types of neuron-like oscillators (Hodgkin-Huxley model and Rulkov map) were investigated. We compare the results obtained in a discrete neuron model (Rulkov model) and a continuoustime model (Hodgkin-Huxley). We show that in the case of a discrete neuron model, the periodic dynamics is manifested in the alternate excitation of various neural ensembles. In the case of the continuous-time model, periodic modulation of the synchronization index of neural ensembles is observed.

\section{Mathematical Model}

The time evolution of the transmembrane potential of the HH neurons is given by [Hodgkin and Huxley, 1952]:

$$
C_{m} \frac{d V_{i}}{d t}=-g_{N a}^{\max } m_{i}^{3} h_{i}\left(V_{i}-V_{N a}\right)-g_{K}^{\max } n_{i}^{4}\left(V_{i}-V_{K}\right)-
$$$$
-g_{L}^{\max }\left(V_{i}-V_{L}\right)+I_{i}^{e x}+I_{i}^{\text {syn }}
$$

where $C_{m}=1 \mu \mathrm{F} / \mathrm{cm}^{3}$ is the capacity of cell membrane, $I_{i}^{e x}$ is an external bias current injected into a neuron in the network, $V_{i}$ is the membrane potential of $i$-th neuron, $i=1, \ldots, N, g_{N a}^{\max }=120 \mathrm{mS} / \mathrm{cm}^{2}, g_{K}^{\max }=$ $36 \mathrm{mS} / \mathrm{cm}^{2}$ and $g_{L}^{\max }=0.3 \mathrm{mS} / \mathrm{cm}^{2}$ receptively denote the maximal sodium, potassium and leakage conductance when all ion channels are open. $V_{N a}=50 \mathrm{mV}$, $V_{K}=-77 m V$ and $V_{L}=-54.4 m V$ are the reversal potentials for sodium, potassium and leak channels respectively. $m, n$ and $h$ represent the mean ratios of the open gates of the specific ion channels. $n^{4}$ and $m^{3} h$ are the mean portions of the open potassium and sodium ion channels within a membrane patch. The dynamics of gating variables $(x=m, n, h)$ depending on rate functions $\alpha_{x}(V)$ and $\beta_{x}(V)$ are given [Pankratova and Polovinkin, 2005]:

$$
\frac{d x_{i}}{d t}=\alpha_{x_{i}}\left(V_{i}\right)\left(1-x_{i}\right)-\beta_{x_{i}}\left(V_{i}\right) x_{i}, \quad x=m, n, h
$$

$I_{i}^{s y n}$ is the total synaptic current received by neuron $i$. We consider coupling via chemical synapses. The synaptic current takes the form [White et al., 2000]

$$
I_{i}^{\text {syn }}=\sum_{j \in \text { neigh }(i)} g_{c} \alpha\left(t-t_{0}^{j}\right)\left(E_{\text {rev }}-V_{i}\right)
$$

where the alpha function $\alpha(t)$ describes the temporal evolution of the synaptic conductance, $g_{c}$ is the maximal conductance of the synaptic channel and $t_{0}^{j}$ is the time at which presynaptic neuron $j$ fires. We suppose $\alpha(t)=\mathrm{e}^{-t / \tau_{s y n}} \Theta(t)$, there $\Theta(t)$ is the Heaviside step function and $\tau_{\text {syn }}=3 \mathrm{~ms}$.

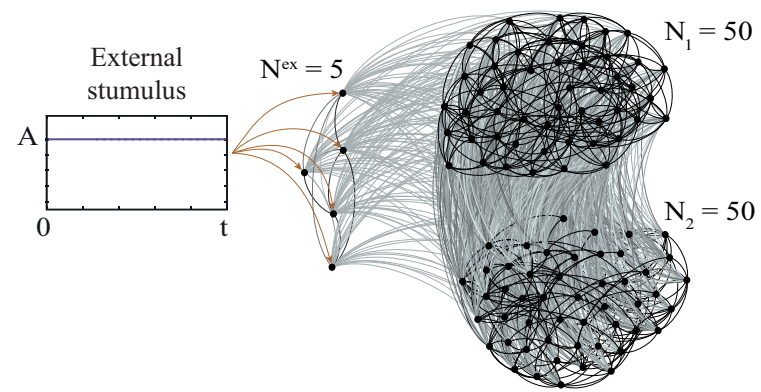

Figure 1. Network model. The external stimulus with amplitude $A$ is applied to the first subnetwork of $N^{e x}=5$ neurons. The subnetwork is connected with the two other subnetworks of $N_{1}=N_{2}=50$ neurons by one-directional excitatory couplings. The subnetworks $N_{1}$ and $N_{2}$ are connected with each other by two-directional inhibitory couplings.

Each Rulkov map [Rulkov et al., 2004] is described by the following system of equations

$$
\begin{aligned}
& x_{n+1}=f\left(x_{n}, x_{n-1}, y_{n}+\beta_{n}\right), \\
& y_{n+1}=y_{n}-\mu\left(x_{n}+1\right)+\mu \sigma+\mu \sigma_{n}+\mu,
\end{aligned}
$$

where $x$ and $y$ are fast and slow variables associated with membrane potential and gating variables, respectively, $\alpha, \sigma$ and $\mu \in(0,1]$ are parameters which regulate the system dynamics, $f$ is a piecewise function defined as

$$
\begin{aligned}
& f\left(x_{n}, x_{n-1}, y_{n}\right)= \\
& \begin{cases}\alpha /\left(1-x_{n}\right)+y_{n}, & \text { if } x_{n} \leq 0, \\
\alpha+y_{n}, & \text { if } 0<x_{n}<\alpha+y_{n} \& x_{n-1} \leq 0, \\
-1, & \text { if } x_{n} \geq \alpha+y_{n} \text { or } x_{n-1}>0,\end{cases}
\end{aligned}
$$

constructed in a way to reproduce different regimes of neuron-like activity, such as spiking, bursting and silent regimes. Here, $\beta_{n}$ and $\sigma_{n}$ are parameters related to external stimuli and defined as

$$
\begin{aligned}
& \beta_{n}=\beta^{e} I_{n}^{\text {ext }}+\beta^{\text {syn }} I_{n}^{\text {syn }}, \\
& \sigma_{n}=\sigma^{e} I_{n}^{\text {ext }}+\sigma^{\text {syn }} I_{n}^{\text {syn }},
\end{aligned}
$$

where $\beta^{e}$ and $\sigma^{e}$ are coefficients used to balance the effect of external current $I_{n}^{\text {ext }}$ defined as

$$
I_{n}^{e x p}= \begin{cases}0, & n<t_{s}, \\ A, & n \geq t_{s},\end{cases}
$$

$\beta^{s y n}$ and $\sigma^{\text {syn }}$ are coefficients of chemical synaptic coupling [Hu and Cao, 2016], and $I_{n}^{s y n}$ is a synaptic current 


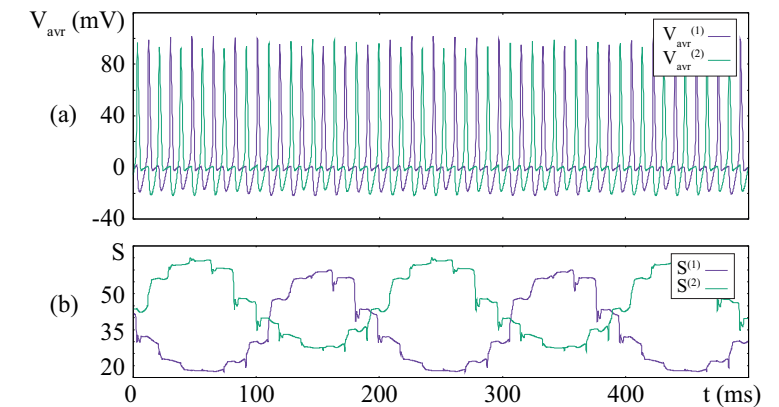

Figure 2. (a) Time evolution of $V_{a v r}^{(1)}$ and $V_{a v r}^{(2)}$ averaged over all neurons of subnetworks $N_{1}$ and $N_{2}$ respectively. (b) Time evolution of synchronization indexes $S^{(1)}$ and $S^{(2)}$ for the same subnetworks. Network topology for subnetworks $N_{1}$ and $N_{2}$ is "all-to-all", probability of coupling between two neurons of the subnetworks is $100 \%$, strength of coupling between $N_{1}$ and $N_{2} g_{c}=-0.03$.

given as

$I_{n+1}^{s y n}=\gamma I_{n}^{s y n}-g_{s y n} *$ $\begin{cases}\left(x_{n}^{\text {post }}-x_{r p}\right) /\left(1+e^{-k\left(x_{n}^{\text {post }}-\theta\right)}\right), & x_{n}^{\text {pre }} \geq \alpha+y_{n}^{\text {pre }} \\ & +\beta_{n}^{\text {pre }} \\ 0, & \text { otherwise }\end{cases}$

where $g_{\text {syn }} \geq 0$ is the strength of synaptic coupling, $\theta=-1.55$ and $k=50$ are synaptic parameters which stand for the synaptic threshold behavior. The super indices pre and post refer, respectively, to the presynaptic and postsynaptic variables, $\gamma \in[0,1]$ is the synaptic relaxation time defining a portion of synaptic current preserved in the next iteration, and $x_{r p}$ is a reversal potential determining the type of synapse, inhibitory or excitatory. The parameter values are chosen so that uncoupled neurons are in a resting state, namely, $\alpha=3.65, \sigma=0.06$ and $\mu=0.0005$. We also assume $\beta^{e}=0.133, \sigma^{e}=1.0$, $\beta^{\text {syn }}=0.1, \sigma^{\text {syn }}=0.5$.

\section{Results}

We investigate dynamics of a network consisting of 3 subnetworks (Fig. 1). The external stimulus with amplitude $A$ is applied to the first subnetwork of $N^{e x}=5$ neurons. The role of this subnetwork is to translate a constant input signal into a spike sequence; an increase in the amplitude of the signal leads to an increase in the frequency of generation of spikes, thus modulating the amplitude with a frequency. Inside it all neurons are connected with each other by two-directional excitatory couplings, coupling strength is chosen randomly from the range [0;0.15]. The subnetwork $N^{e x}$ is connected with the two other subnetworks of $N_{1}=N_{2}=50$ neurons by one-directional excitatory couplings with $30 \%$ probability. The subnetworks $N_{1}$ and $N_{2}$ are connected with each other by two-directional inhibitory couplings with coupling strength $g_{c}$ and probability $p$.

First, we choose Hodgkin-Huxley neuron model as an element of the network. For this case the external stimu- lus with amplitude $A=9 \mu \mathrm{A} / \mathrm{cm}^{2}$ is applied to the first subnetwork that corresponds to the regime of continuous spikes generation, and the current with amplitude $A=6 \mu \mathrm{A} / \mathrm{cm}^{2}$ s applied to $N_{1}$ and $N_{2}$ subnetworks corresponding to "silent" regime when all neurons do not generate spikes. We analyze the dynamics of the membrane potentials averaged over $N_{1}$ and $N_{2}$ subnetwork: $V_{a v r}=\sum_{i=1}^{N} \frac{V_{i}}{N}$, where $N$ is a number of neurons in the subnetwork.

We consider three different network's topologies: (1) "all-to-all", (2) "small-world", and (3) "random topology". For "all-to-all" topology coupling strength is chosen randomly from the range $[0 ; 0.15]$. For $p=100 \%$, $g_{c}=-0.03$ we find a phenomenon of $N_{1}$ and $N_{2}$ subnetworks generating spikes in antiphase. So we can say that two subnetworks are synchronized to each other with a time lag of a half of a period [Blekhman et al., 1997]. Moreover, in the dynamics of membrane potentials averaged over each subnetwork, low-frequency oscillations of the amplitude were observed, which, in turn, were also in antiphase [see Fig. 2 (a)]. In order to investigate it we calculate synchronization index as follows [Wang et al., 2009; Sausedo-Solorio and Pisarchik, 2017]:

$$
S=\sqrt{\frac{1}{T} \sum_{n=1}^{N} \xi_{n}}
$$

where $\xi_{n}$ is the standard deviation given as

$$
\xi_{n}=\frac{1}{N} \sum_{i=1}^{N}\left(x_{n}^{(i)}\right)^{2}-\left(\frac{1}{N} \sum_{i=1}^{N} x_{n}^{(i)}\right)^{2} .
$$

where $T$ is a number of iterations, $N$ is a number of neurons in the subnetwork. The smaller $S$, the better the

(a)

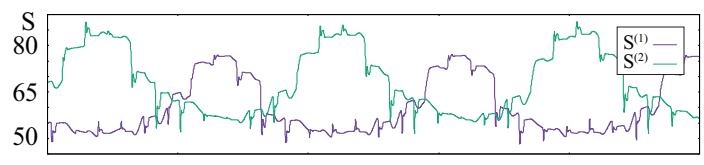

(b) 90

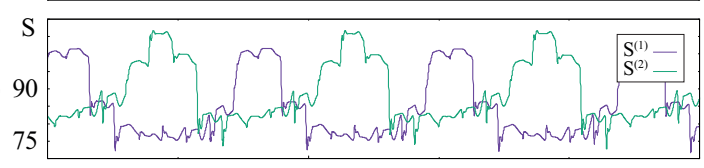

(c) 60

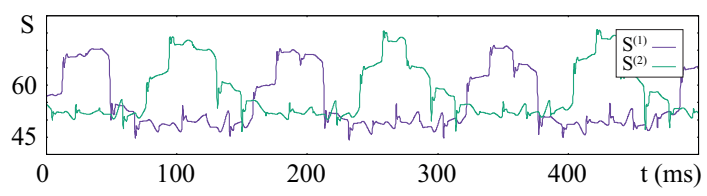

Figure 3. Time evolution of synchronization indexes $S^{(1)}$ and $S^{(2)}$ for the subnetworks $N_{1}$ and $N_{2}$ for different topologies: (a) "all-toall", strength of coupling between the elements inside the subnetworks is chosen randomly from the range [0;0.15]; (b) "small-world"; (c) "random topology". For all cases probability of coupling between two neurons of the subnetworks is $30 \%$, strength of coupling between $N_{1}$ and $N_{2} g_{c}=-0.09$. 
(a) 50

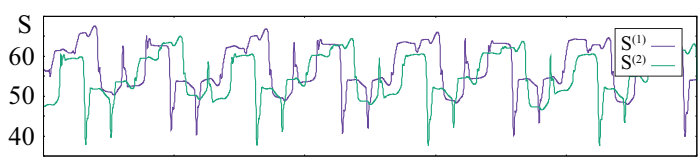

(b)

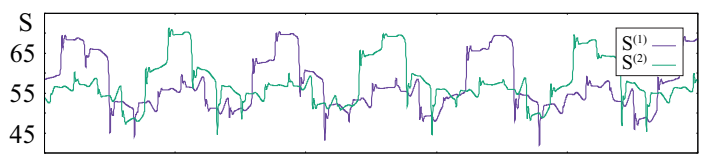

(c)

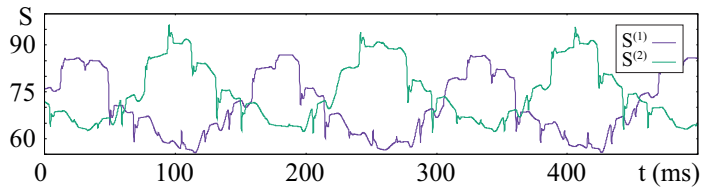

Figure 4. Time evolution of synchronization indexes $S^{(1)}$ and $S^{(2)}$ for the subnetworks $N_{1}$ and $N_{2}$ respectively for "small-world" topology for different values of strength of coupling between $N_{1}$ and $N_{2}$ : (a) $g_{c}=-0.06$, (b) $g_{c}=-0.08$, (c) $g_{c}=-0.1$. For all cases probability of coupling between two neurons of the subnetworks is $30 \%$.

synchronization; $S=0$ means complete synchronization.

Fig. 3 (b) illustrates time evolution of synchronization indexes $S^{(1)}$ and $S^{(2)}$ of the averaged signals $V_{a v r}^{(1)}$ and $V_{a v r}^{(2)}$. One can see that the synchronization indexes oscillate in antiphase. Thus, the subnetworks, interacting with each other, in turn process the information arriving to them, while maintaining the overall activity constant.

If we make the probability of coupling between neurons of $N_{1}$ and $N_{2}$ subnetworks $p=30 \%$, we still can observe synchronization indexes oscillating in antiphase [Fig. 3 (a)]. But $g_{c}$ must be bigger to make the input synaptic current the same as number of inpus couplings is decreased. Comparing Fig. 3 (a) and 3 (b) one can see that the oscillation amplitude decreases but $S$ is bigger for all considered time.

Nest, we investigate how different network topologies influence on the subnetworks interaction. In addition to the already studied "all-to-all" topology, we consider "small world" (SW) and "random topology". SW network is generated using the Watts-Strogatz model [Watts and Strogatz, 1998] with parameters $\beta=0.3$ and $K=$ 5 . The parameter $\beta$ is the probability for a particular link in the initially regular topology to be randomly rewired, and $K$ is the mean degree. The completely random network is generated as a limit case of the Watts-Strogatz model with $\beta=1$, meaning that all links of the initially regular topology are randomly rewired. Figs. $3($ b,c) illustrate time evolution of synchronization indexes for the subnetworks $N_{1}$ and $N_{2}$ for $\mathrm{SW}$ and random topologies respectively. One can see that synchronization indexes oscillate in antiphase for all considered topologies. Oscillation amplitude of $S$ is the same for all of them, the oscillation period is the largest for "all-to-all" topology, $S$ takes its highest values for the "small world", and the smallest for the random one.

For further research, we choose SW topology was chosen due to the fact that this topology determines neuronal coupling on an anatomical level [Muldoon et al., 2016; Bassett and Bullmore, 2006]. We investigate influence of strength of coupling $g_{c}$ between $N_{1}$ and $N_{2}$ subnetworks on synchronization of all neurons inside each of them. Fig. 4 illustrates time evolution of synchronization indexes for three different values of $g_{c}$. For small coupling strength (a) synchronization indexes oscillate in phase. Increasing $g_{c}$ leads to indexes start to oscillate in antiphase, but oscillation amplitude remains the same (b). If we continue to increase $g_{c}$ oscillation amplitude and synchronization indexes increases (c).

Next, we choose Rulkov map as an element of the network. For this case the external stimulus with amplitude $A=2$ is applied to the first subnetwork that corresponds to the regime of continuous spikes generation, and no current is applied to $N_{1}$ and $N_{2}$ subnetworks. The dynamics for this network differs from one demonstrated by the Hodgkin-Huxley neurons system. For a network of $\mathrm{HH}$ neurons both subnetworks alternately generated spikes [Fig. 2 (a)], but for a network of Rulkov maps the dynamics of the averaged signal of each subnetwork is characterized by alternating periods of "silence" of the network with periods of continuous generation of spikes (Fig. 5), and if one subnetwork is in "silent" regime, then the second one is in spike generation regime, and vice versa. Thus, two interacting subnetworks demonstrate antiphase dynamics, while maintaining the overall dynamics constant. The figure illustrates that as soon as one subnetwork activates, the second subnetwork immediately deactivates.

\section{Conclusion}

We have investigated of a neural network included several subnetworks. The first subnetwork was used to translate a constant-amplitude signal into a spike sequence. The first subnetwork was connected to two large interconnected subnetworks by excitatory couplings. It was shown that in the presence of inhibitory coupling between two large subnetworks, the neuronal ensembles demonstrate antiphase dynamics. Various connectivity topologies ("all-to-all", "small-world" and completely random) and various types of neuron-like oscillators (Hodgkin-Huxley model and Rulkov map) were investigated. We have compared the results obtained in a

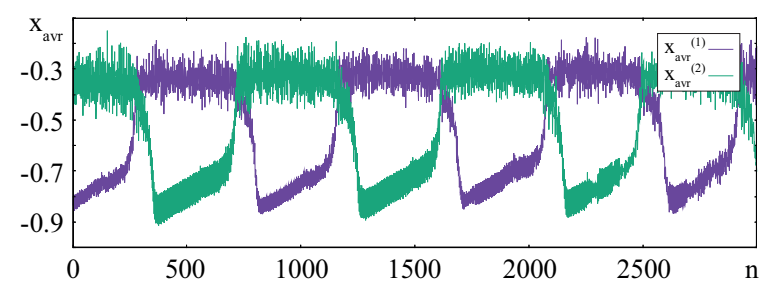

Figure 5. Time evolution of $x_{\text {avr }}^{(1)}$ and $x_{\text {avr }}^{(2)}$ averaged over all neurons of subnetworks $N_{1}$ and $N_{2}$ respectively for Rulkov map network. $x_{r p}=-2.5$ for the couplings between $N_{1}$ and $N_{2}$, $g_{c}=-0.05$. 
discrete neuron model (Rulkov model) and a continuoustime model (Hodgkin-Huxley). It is shown that in the case of a discrete neuron model, the periodic dynamics is manifested in the alternate excitation of various neural ensembles. In the case of the continuous-time model, periodic modulation of the synchronization index of neural ensembles is observed. This is manifested in low-frequency modulation of neural activity. According to the literature, low-frequency modulation of spike activity of neurons occurs due to cortical activity rhythms (for example, theta, alpha, and beta), which are recorded non-invasively using EEG. The influence of strength of coupling between large subnetworks on synchronization of all neurons inside each of them was investigated.

The constructed theoretical model of a network of neuron-like elements can be used to explain the effect of the oscillatory dynamics of cognitive performance. Given that cognitive performance is determined by the degree of involvement of neural populations of the cerebral cortex in information processing, it can be assumed that the cognitive load is constantly redistributed between different populations of the cerebral cortex.

\section{Acknowledgements}

This work was supported by the Russian Foundation for Basic Research (Grant 18-32-20129).

\section{References}

Andreev, A., Frolov, N., Pisarchik, A., and Hramov, A. (2019). Chimera state in complex networks of bistable hodgkin-huxley neurons. Physical Review E, 100 (2), pp. 022224.

Andreev, A., Makarov, V., Runnova, A., and Hramov, A. (2017). Coherent resonance in neuron ensemble with electrical couplings. Cybernetics and Physics, 6(3), pp. 135-138.

Bassett, D. S. and Bullmore, E. (2006). Small-world brain networks. The neuroscientist, 12 (6), pp. 512523.

Blekhman, I., Fradkov, A., Nijmeijer, H., and Pogromsky, A. Y. (1997). On self-synchronization and controlled synchronization. Systems \& Control Letters, 31 (5), pp. 299-305.

Boccaletti, S., Latora, V., Moreno, Y., Chavez, M., and Hwang, D.-U. (2006). Complex networks: Structure and dynamics. Physics reports, 424 (4-5), pp. 175308.

Bolotov, M., Osipov, G., and Pikovsky, A. (2016). Marginal chimera state at cross-frequency locking of pulse-coupled neural networks. Physical Review E, 93 (3), pp. 032202.

Bullmore, E. and Sporns, O. (2009). Complex brain networks: graph theoretical analysis of structural and functional systems. Nature reviews neuroscience, 10 (3), pp. 186.

Buzsáki, G. (2004). Large-scale recording of neuronal ensembles. Nature neuroscience, 7 (5), pp. 446.
Buzsáki, G. and Draguhn, A. (2004). Neuronal oscillations in cortical networks. science, 304 (5679), pp. 1926-1929.

Campbell, I. G. (2009). Eeg recording and analysis for sleep research. Current protocols in neuroscience, 49 (1), pp. 10-2.

Cavanna, A. E. and Monaco, F. (2009). Brain mechanisms of altered conscious states during epileptic seizures. Nature Reviews Neurology, 5 (5), pp. 267.

Chholak, P., Niso, G., Maksimenko, V. A., Kurkin, S. A., Frolov, N. S., Pitsik, E. N., Hramov, A. E., and Pisarchik, A. N. (2019). Visual and kinesthetic modes affect motor imagery classification in untrained subjects. Scientific reports, 9 (1), pp. 1-12.

Gao, J., Li, D., and Havlin, S. (2014). From a single network to a network of networks. National Science Review, 1 (3), pp. 346-356.

Gómez-Gardenes, J., Moreno, Y., and Arenas, A. (2007). Paths to synchronization on complex networks. Physical review letters, 98 (3), pp. 034101.

Gómez-Gardenes, J., Moreno, Y., and Arenas, A. (2011). Evolution of microscopic and mesoscopic synchronized patterns in complex networks. Chaos: An Interdisciplinary Journal of Nonlinear Science, 21(1), pp. 016105.

Haenschel, C., Vernon, D. J., Dwivedi, P., Gruzelier, J. H., and Baldeweg, T. (2005). Event-related brain potential correlates of human auditory sensory memorytrace formation. Journal of Neuroscience, 25(45), pp. 10494-10501.

Hodgkin, A. L. and Huxley, A. F. (1952). A quantitative description of membrane current and its application to conduction and excitation in nerve. The Journal of physiology, 117 (4), pp. 500-544.

Hu, D. and Cao, H. (2016). Stability and synchronization of coupled rulkov map-based neurons with chemical synapses. Communications in Nonlinear Science and Numerical Simulation, 35, pp. 105-122.

Jiang, L.-L. and Perc, M. (2013). Spreading of cooperative behaviour across interdependent groups. Scientific reports, 3, pp. 2483.

Klinshov, V., Lücken, L., Shchapin, D., Nekorkin, V., and Yanchuk, S. (2015). Multistable jittering in oscillators with pulsatile delayed feedback. Physical review letters, 114 (17), pp. 178103.

Klinshov, V. V., Teramae, J.-n., Nekorkin, V. I., and Fukai, T. (2014). Dense neuron clustering explains connectivity statistics in cortical microcircuits. PloS one, 9 (4), pp. e94292.

Maksimenko, V., Badarin, A., Nedaivozov, V., Kirsanov, D., and Hramov, A. (2018a). Brain-computer interface on the basis of eeg system encephalan. In Saratov Fall Meeting 2017: Laser Physics and Photonics XVIII; and Computational Biophysics and Analysis of Biomedical Data IV, vol. 10717, International Society for Optics and Photonics, p. 107171R.

Maksimenko, V. A., Hramov, A. E., Frolov, N. S., 
Lüttjohann, A., Nedaivozov, V. O., Grubov, V. V., Runnova, A. E., Makarov, V. V., Kurths, J., and Pisarchik, A. N. (2018b). Increasing human performance by sharing cognitive load using brain-to-brain interface. Frontiers in neuroscience, 12.

Maslennikov, O. V. and Nekorkin, V. I. (2017). Adaptive dynamical networks. Physics-Uspekhi, 60 (7), pp. 694.

Moreno, Y. and Pacheco, A. F. (2004). Synchronization of kuramoto oscillators in scale-free networks. EPL (Europhysics Letters), 68 (4), pp. 603.

Muldoon, S. F., Bridgeford, E. W., and Bassett, D. S. (2016). Small-world propensity and weighted brain networks. Scientific reports, 6, pp. 22057.

Palmeri, T. J. and Gauthier, I. (2004). Visual object understanding. Nature Reviews Neuroscience, 5 (4), pp. 291.

Pankratova, E. V. and Polovinkin, A. V. (2005). Resonant activation in a stochastic hodgkin-huxley model: interplay between noise and suprathreshold driving effects. European Physical Journal B, 45, pp. 391.

Rulkov, N. F., Timofeev, I., and Bazhenov, M. (2004). Oscillations in large-scale cortical networks: mapbased model. Journal of computational neuroscience,
17 (2), pp. 203-223.

Sausedo-Solorio, J. and Pisarchik, A. (2017). Synchronization in network motifs of delay-coupled mapbased neurons. The European Physical Journal Special Topics, 226 (9), pp. 1911-1920.

Valencia, M., Martinerie, J., Dupont, S., and Chavez, M. (2008). Dynamic small-world behavior in functional brain networks unveiled by an event-related networks approach. Physical Review E, 77 (5), pp. 050905.

Wang, Q., Perc, M., Duan, Z., and Chen, G. (2009). Synchronization transitions on scale-free neuronal networks due to finite information transmission delays. Physical Review E, 80 (2), pp. 026206.

Watts, D. J. and Strogatz, S. H. (1998). Collective dynamics of 'small-world' networks. nature, 393 (6684), pp. 440.

White, J. A., Rubinstein, J. T., and Kay, A. R. (2000). Channel noise in neurons. Trends in Neurosciences, 261, pp. 83-92.

Yun, K., Watanabe, K., and Shimojo, S. (2012). Interpersonal body and neural synchronization as a marker of implicit social interaction. Scientific reports, 2, pp. 959. 